\title{
Vocal cord paralysis due to ectopic parathyroid adenoma and function recovery: a case report and review of the literature
}

\author{
Teng Zhao*, Yunhui Xin*, Hong Shen, Xing Liu, Jiacheng Wang, Qian Wang and Bojun Wei \\ Department of Thyroid and Neck Surgery, Beijing Chaoyang Hospital, Capital Medical University, No. 8 Gongren Tiyuchang \\ Nanlu, Chaoyang District, Beijing, 100020, China
}

\begin{abstract}
Ectopic parathyroid adenomas (PAs) can occur in numerous locations and are thought to be the cause of a significant portion of failed primary surgery for hyperparathyroidism. PA is a rare cause of hoarseness, which may be harbingers of a malignant process. Here, we describe an unusual case of an ectopic PA in the carotid sheath presenting as unilateral vocal cord paralysis (VCP). A 49-year-old lady presented with a 1-week history of hoarseness, irritating cough and shortness of breath. Fibreoptic laryngoscopy revealed left VCP. Ultrasound and computed tomography of the neck demonstrated a mass in the carotid sheath. Laboratory investigations revealed hypercalcemia $(3.10 \mathrm{mmol} / \mathrm{L})$, hypophosphatemia $(0.81 \mathrm{mmol} / \mathrm{L})$ and elevated intact parathyroid hormone (iPTH) level $(381.6 \mathrm{pg} / \mathrm{mL})$, despite of a negative ${ }^{99 \mathrm{~m}} \mathrm{Tc}$-sestamibi scan. After more rigorous tests, the ectopic tumor adjacent to the left vagus nerve was successfully resected, with subsequent histopathological confirmation of PA. The patient eventually got a normal iPTH level and serum calcium postoperatively, and regular voice function was also regained 4 months after surgery. This case emphasizes the importance of broad differential diagnosis and thorough workup. Although most patients with PA present with hypercalcemia, this disease entity also need to be considered in the differentials of neck masses and VCP.
\end{abstract}

Key words: Ectopic, Parathyroid adenoma, Vocal cord paralysis, Vagus nerve, Case report

THE INCIDENCE AND PREVALENCE of primary hyperparathyroidism (PHPT) have increased globally during the past several decades, with the majority being secondary to parathyroid adenoma (PA) [1]. PAs typically present with symptoms of hypercalcemia, manifested by polyuria, polydipsia, nephrolithiasis, bone pain, constipation, anorexia, vomiting, arrhythmias, and altered mental status, with asymptomatic disorders becoming more common [2]. Vocal cord paralysis (VCP), which may be harbingers of malignancy, however, has rarely been associated with PA $[2,3]$.

Ectopic PAs, accounting for $4 \%$ to $16 \%$ of patients with hyperparathyroidism, are thought to be the cause of a significant portion of failed primary surgery for hyperparathyroidism $[4,5]$. They can occur in numerous anatomic locations including the mediastinum, the retro/

Submitted Aug. 11, 2019; Accepted Oct. 5, 2019 as EJ19-0349

Released online in J-STAGE as advance publication Nov. 2, 2019

Correspondence to: Bojun Wei, M.D., Ph.D., Department of Thyroid and Neck Surgery, Beijing Chaoyang Hospital, Capital Medical University, No. 8 Gongren Tiyuchang Nanlu, Chaoyang District, Beijing, 100020, China.

E-mail: weibojun1015@126.com

*Teng Zhao and Yunhui Xin contributed equally as first authors. paraesophageal space, intrathymic or intrathyroidal sites, as well as the carotid sheath $[6,7]$. Here, we describe a rare case of ectopic PA in the carotid sheath presenting as unilateral vocal cord paralysis (VCP) to discuss implications for diagnosis and management.

\section{Case Presentation}

A 49-year-old lady presented with a 1-week history of hoarseness, irritating cough and shortness of breath. History-taking elicited no other associated symptoms. There was no family history of thyroid/parathyroid disorders or history of previous radiation. Physical examination of her neck was unremarkable. Fibreoptic laryngoscopy revealed left vocal cord immobility, with no intrinsic vocal cord lesion identified, which was consistent with left recurrent laryngeal nerve (RLN) or vagus nerve paralysis (Fig. 1a, b).

Neck ultrasound revealed a cystic mass with septation of dimension $4.7 \mathrm{~cm} \times 2.2 \mathrm{~cm}$ between the left common carotid artery and the left internal jugular vein, with the suspicion of neurogenic tumor (Fig. 2a). A hypoechoic nodule (TI-RADS 3 category) of dimension $0.4 \mathrm{~cm} \times 0.2$ $\mathrm{cm}$ was also noted on the left thyroid lobe and adjacent 


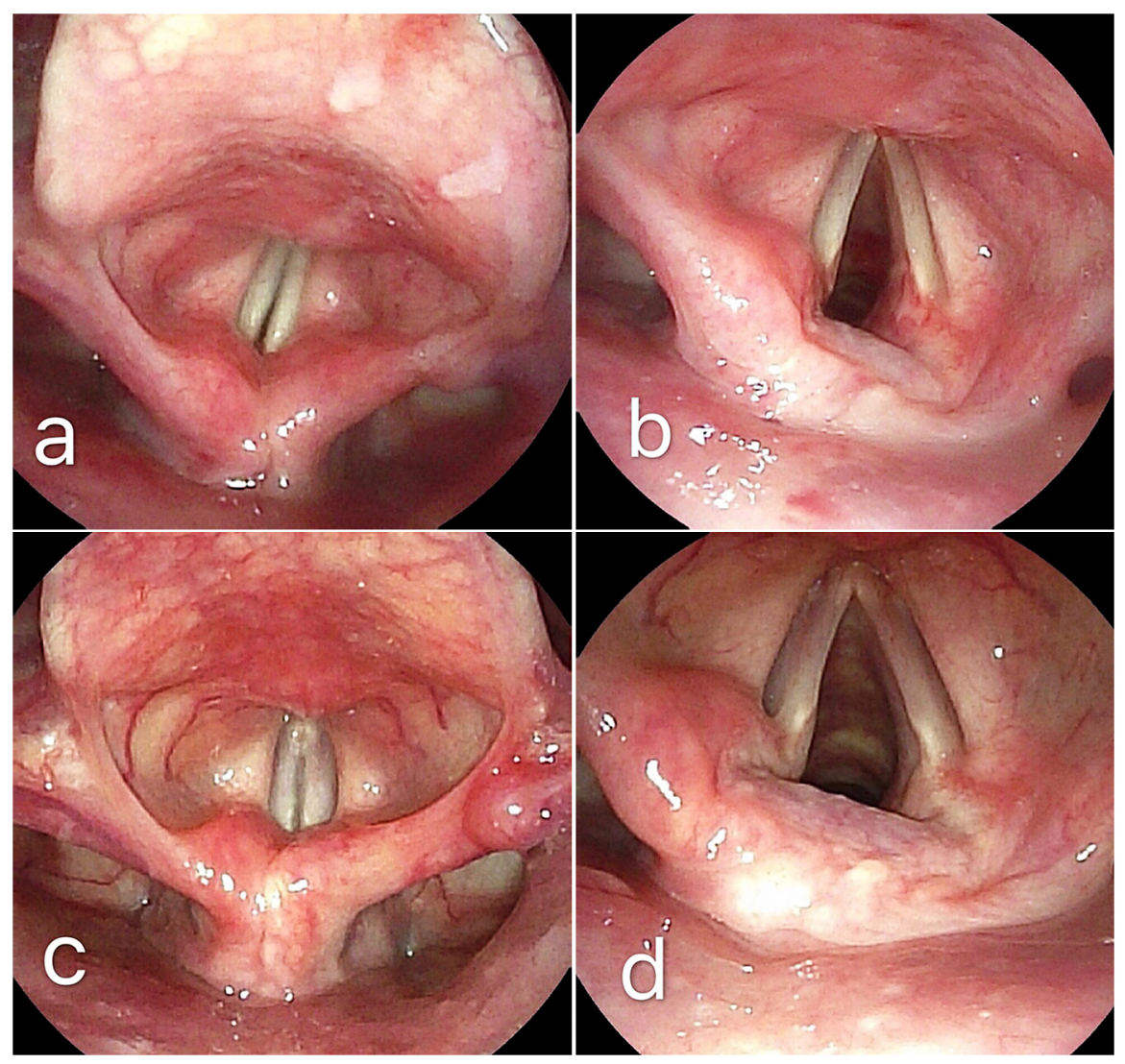

Fig. 1 Laryngoscopy showing paretic left vocal cord at presentation and return of function postoperatively. Preoperative laryngoscopy showed left vocal cord immobility, with no intrinsic vocal cord lesion identified (a, b). Follow-up showed marked improvement in left vocal movement, and vocal cords closed more firmly after operation (c, d).
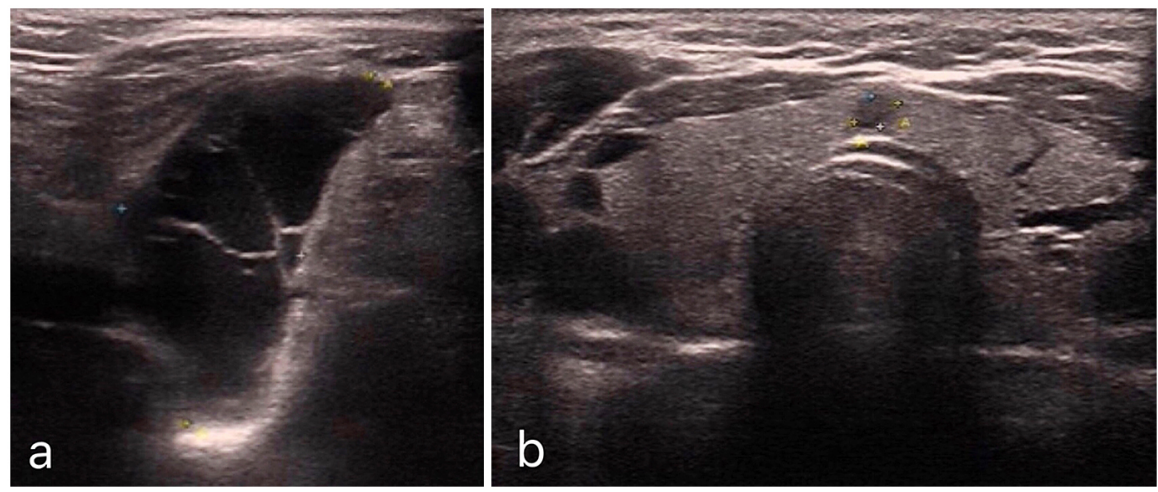

Fig. 2 Neck ultrasound images showing a cystic mass in the carotid sheath as well as a thyroid nodule. The cystic mass with septation measures $4.7 \mathrm{~cm} \times 2.2 \mathrm{~cm}$ (a). The hypoechoic thyroid nodule (TI-RADS 3 category) localized on the left lobe and adjacent to the isthmus measures $0.4 \mathrm{~cm} \times 0.2 \mathrm{~cm}(\mathrm{~b})$.

to the isthmus (Fig. 2b). Furthermore, an additional hyperechoic mass of dimension $0.6 \mathrm{~cm} \times 0.5 \mathrm{~cm}$ caudal to the lower pole of the left thyroid lobe was detected. Lateral cervical lymph nodes were not enlarged. Further investigations included a contrast computed tomography (CT) of the neck showing a solid and cystic lesion of 5.0 $\mathrm{cm}$ in diameter in the carotid sheath. Similarly, the appearance was presumed to be a neurogenic tumor with cystic degeneration (Fig. 3).

The patient was admitted for surgery, and hypercalcemia $(3.10 \mathrm{mmol} / \mathrm{L})$ accompanied with hypophosphatemia $(0.81 \mathrm{mmol} / \mathrm{L})$ was accidentally discovered during routine preoperative assessment. Other serum blood tests were within normal limits, despite of an elevated intact 


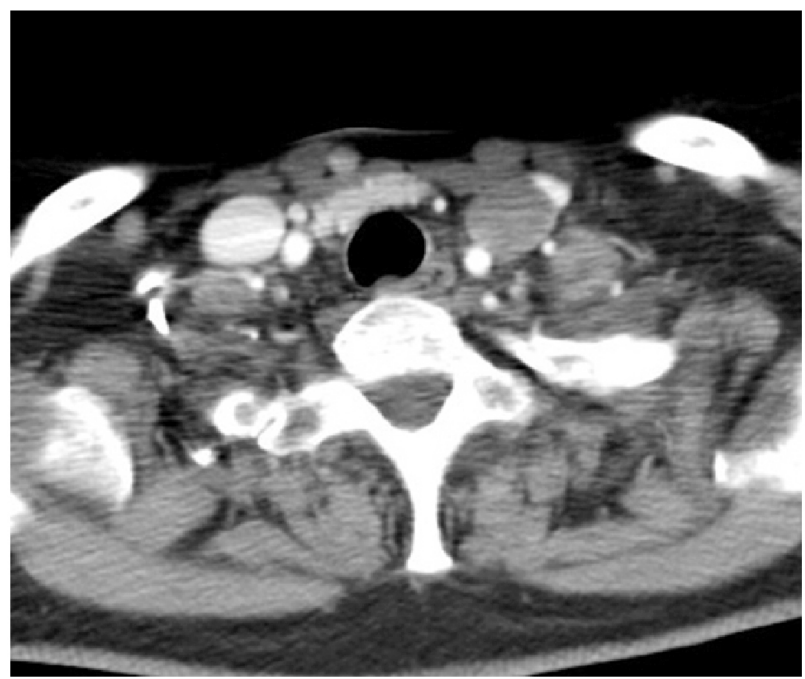

Fig. 3 Axial computed tomography (CT) scan of the neck shows a solid and cystic lesion in the carotid sheath. The lesion, measuring $5.0 \mathrm{~cm}$ in diameter, is located between the left common carotid artery and the left internal jugular vein.

parathyroid hormone (iPTH) level $(381.6 \mathrm{pg} / \mathrm{mL})$ as well as a low 25 -hydroxyvitamin D level $(5.57 \mathrm{ng} / \mathrm{mL})$. However, further ${ }^{99 \mathrm{~m}} \mathrm{Tc}$-sestamibi scan did not reveal any abnormality. Abdominal ultrasound was also negative for nephrolithiasis. Bone mineral density assessment was not further scheduled before surgery given that the patient didn't suffer any bone pain or fragility fracture.

After more rigorous tests, an exploratory parathyroidectomy and isthmusectomy with intraoperative PTH (IOPTH) monitoring and intraoperative neuromonitoring (IONM) was then arranged under general anesthetic. Blood samples were taken from bilateral internal jugular veins and a dedicated peripheral arterial line after isthmusectomy (pre-excision baseline), and then 5 minutes after excision of the lesion, which demonstrated a higher baseline iPTH level in the left internal jugular vein $(1,101.4 \mathrm{pg} / \mathrm{mL})$ than in right $(387.1 \mathrm{pg} / \mathrm{mL})$. Intraopera- tive findings showed a predominantly cystic parathyroid mass of dimension $5.5 \mathrm{~cm} \times 2.5 \mathrm{~cm} \times 2.0 \mathrm{~cm}$ with adherence to, but no invasion of, the left vagus nerve (Fig. $4 a$ ). No visual change in the nerve was noted. With a stimulation level of $2.0 \mathrm{~mA}$ and an event threshold of $50 \mu \mathrm{V}$, electromyographic (EMG) responses could be elicited from the RLN and the distal portion of vagus nerve (inferior to the mass), though with decreased amplitudes (95 $\mu \mathrm{V}$ and $74 \mu \mathrm{V}$, respectively), while the EMG waveform can not be observed from the proximal portion of vagus nerve (superior to the mass). The mass was carefully delineated from the vagus nerve, and weighed $1.93 \mathrm{~g}$. The vagus nerve appeared fully intact after the mass was removed (Fig. 4b). The specimen had a multi-cystic, haemorrhagic appearance with well-defined borders, and contained haemorrhagic fluid. The level of iPTH in the obtained cystic fluid was more than $1,900 \mathrm{pg} / \mathrm{mL}$, and IOPTH monitoring demonstrated a marked decline in peripheral arterial blood, from $349.4 \mathrm{pg} / \mathrm{mL}$ prior to excision to $21.7 \mathrm{pg} / \mathrm{mL}$ after 5 minutes of excision. Furthermore, the thyroid nodule on the isthmus was also resected with isthmusectomy. The left inferior parathyroid gland, which appeared to be normal, was also examined for histopathology. Frozen section revealed the presence of parathyroid tissue in the ectopic mass in carotid sheath, with final histopathological confirmation of PA with no malignant features (Fig. 5), while the left inferior parathyroid gland was demonstrated to be normal and a benign thyroid nodule was suggested as expected. Her iPTH level and serum calcium decreased to 0.0 $\mathrm{pg} / \mathrm{mL}$ and $2.60 \mathrm{mmol} / \mathrm{L}$ on the right postoperative day, and normalized at $46.9 \mathrm{pg} / \mathrm{mL}$ and $2.14 \mathrm{mmol} / \mathrm{L}$ after 2 days. Follow-up showed normal vocal function and gradually disappeared breathing symptoms 4 months after surgery. Further examination showed marked improvement in left vocal movement, and vocal cords closed more firmly (Fig. 1c, d).
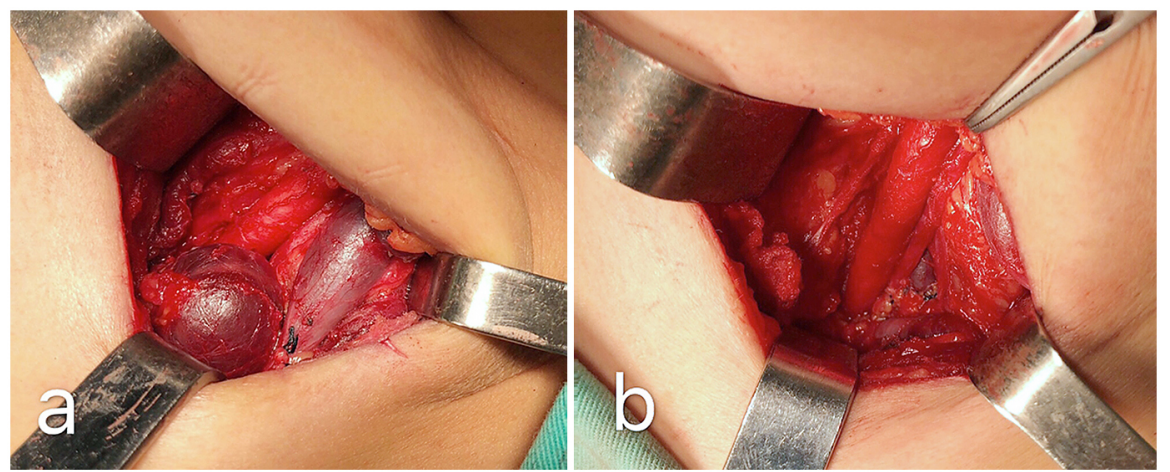

Fig. 4 Intraoperative view of the parathyroid mass and the vagus nerve. The predominantly cystic parathyroid mass was with adherence to, but no invasion of, the left vagus nerve (a). The vagus nerve appeared fully intact after the mass was removed (b). 


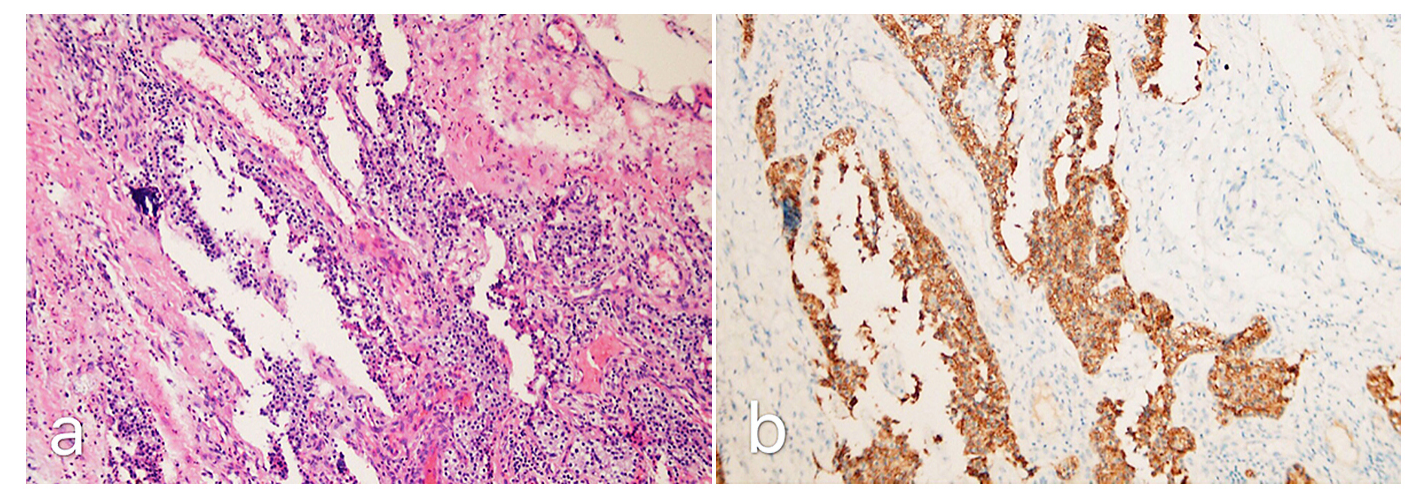

Fig. 5 Histopathology and immunohistochemistry of the ectopic parathyroid tumor in carotid sheath. Pathology of the mass revealed hypercellular parathyroid adenoma with cystic change $(\mathrm{H} \& \mathrm{E}, \times 100)(\mathrm{a})$. Immunohistochemistry showed positivity for parathyroid hormone $(\times 100)(b)$.

\section{Discussion}

PHPT, usually caused by a single functioning PA, is an important clinical issue that affects $0.4 \%$ to $11 \%$ of the population [8-10]. PAs may occur anywhere along the embryologic path following the descent of the parathyroid glands, and the existence of ectopic PA remains a significant hurdle in the surgical management of hyperparathyroidism. The parathyroid glands develop from the third and fourth pharyngeal pouches, while the vagus nerve runs through the fourth pharyngeal arch adjacent to the origin of the parathyroid gland. Ectopic PAs can thus occur in the path of the vagus nerve, which were confirmed in several studies [11-13]. Countless texts have been written about parathyroid exploration in the case of ectopic PAs, however, little attention has been devoted to other unique presentations of PAs. Here we report, for the first time, a patient presenting with sudden-onset VCP and an ectopic parathyroid mass in the carotid sheath, without any typical symptoms of hypercalcemia.

Although PAs do not typically cause VCP, there are several reported scenarios of VCP caused by benign parathyroid disease: (1) direct compression due to the close proximity to the nerve fibers $[3,14],(2)$ rapid expansion of PAs due to spontaneous internal hemorrhage, thereby exhibiting pressure on RLN [15], and (3) stretching of the RLN owing to the pressure effects of a parathyroid cyst [16]. Like the patient in this report, most of these palsies returned to normal postoperatively. We speculate that the sudden-onset VCP in our patient may be related to the direct compression effect to the nerve fibers versus possible history of hemorrhagic changes within the PA, causing rapid nerve compression. Hemorrhagic transformation has been postulated to occur secondary to the vascular nature of PAs [17], as well as secondary to central necrosis of a large PA [18].
The case illustrated several points regarding diagnostic imaging for PHPT. Commonly, ${ }^{99 \mathrm{~m}} \mathrm{Tc}$-sestamibi scan is used as a first-line imaging modality, especially for the efficient investigation of ectopic lesions, with an accuracy of as high as $81 \%[7,19]$. In this patient, however, ${ }^{99 \mathrm{~m}} \mathrm{Tc}$-sestamibi scan was failed to localize this ectopic PA that weighed $1.93 \mathrm{~g}$. The negative result might be related to the cystic degeneration of PA. Therefore, this case highlights the comprehensive utility of ${ }^{99 \mathrm{~m}} \mathrm{Tc}-$ sestamibi scan, CT, ultrasound as well as selective venous sampling to help identify an ectopic lesion and aid in minimizing the extent of surgical dissection.

Previous reports in the literature describe an algorithm for the management of PHPT [20, 21]. Similarly, at our institution, the general approach to minimally invasive parathyroidectomy involves imaging techniques in conjunction with IOPTH and frozen section, which could help rule out multiple-gland disease and predict cure intraoperatively. With a $>50 \%$ drop from baseline and into the normal range within 5 minutes, our patient avoided the risks associated with prolonged and unnecessary bilateral exploration.

In conclusion, PA is a commonly encountered disease entity for the head and neck surgeon, with most discovered on evaluation for hypercalcemia. Our patient presented with VCP and a mass in the carotid sheath, without typical symptoms of hypercalcemia. This case emphasizes the importance of including ectopic PA in the differential diagnosis of a cervical mass accompanied with VCP, given the high ectopic potential of parathyroid glands. Such consideration of broad differential diagnosis is necessary in order to avoid unnecessary extended or re-operative explorations and the associated risks to the patient. 


\section{Disclosure}

None of the authors have any potential conflicts of interest associated with this report.

\section{Acknowledgments}

Not applicable.

\section{References}

1. Yeh MW, Ituarte PH, Zhou HC, Nishimoto S, Liu IL, et al. (2013) Incidence and prevalence of primary hyperparathyroidism in a racially mixed population. J Clin Endocrinol Metab 98: 1122-1129.

2. Givens DJ, Hunt JP, Bentz BG (2013) Uncommon presentations of parathyroid adenoma. Head Neck 35: E265E268.

3. Lee JC, Barkdull GC, Weisman RA (2009) Parathyroid adenoma as a cause of vocal fold paralysis. Arch Otolaryngol Head Neck Surg 135: 712-713.

4. Gaz RD, Doubler PB, Wang CA (1987) The management of 50 unusual hyperfunctioning parathyroid glands. Surgery 102: 949-957.

5. Phitayakorn R, McHenry CR (2006) Incidence and location of ectopic abnormal parathyroid glands. Am J Surg 191: 418-423.

6. Karvounaris DC, Symeonidis N, Triantafyllou A, Flaris N, Sakadamis A (2010) Ectopic parathyroid adenoma located inside the hypoglossal nerve. Head Neck 32: 1273-1276.

7. Okuda I, Nakajima Y, Miura D, Maruno H, Kohno T, et al. (2010) Diagnostic localization of ectopic parathyroid lesions: developmental consideration. Jpn J Radiol 28: 707-713.

8. Kontogeorgos G, Trimpou P, Laine CM, Oleröd G, Lindahl A, et al. (2015) Normocalcaemic, vitamin Dsufficient hyperparathyroidism - high prevalence and low morbidity in the general population: A long-term followup study, the WHO MONICA project, Gothenburg, Sweden. Clin Endocrinol (Oxf) 83: 277-284.

9. Vignali E, Cetani F, Chiavistelli S, Meola A, Saponaro F, et al. (2015) Normocalcemic primary hyperparathyroidism: a survey in a small village of Southern Italy. Endocr Connect 4: 172-178.

10. Cusano NE, Maalouf NM, Wang PY, Zhang C, Cremers SC, et al. (2013) Normocalcemic hyperparathyroidism and hypoparathyroidism in two community-based nonreferral populations. J Clin Endocrinol Metab 98: 2734-2741.

11. Lack EE, Delay S, Linnoila RI (1988) Ectopic parathyroid tissue within the vagus nerve: incidence and possible clini- cal significance. Arch Pathol Lab Med 112: 304-306.

12. Pawlik TM, Richards M, Giordano TJ, Burney R, Thompson N (2001) Identification and management of intravagal parathyroid adenoma. World J Surg 25: 419423.

13. Okuda I, Nakajima Y, Miura D, Maruno H, Kohno T, et al. (2010) Diagnostic localization of ectopic parathyroid lesions: developmental consideration. Jpn J Radiol 28: 707-713.

14. Takimoto T, Okabe $Y$, Ito M, Umeda R (1989) Intravagal parathyroid adenoma. J Laryngol Otol 103: 704-706.

15. Iwasaki K, Usami A, Oida I, Hashimoto Y, Morita H, et al. (1999) Sudden recurrent laryngeal nerve paralysis due to apoplexy of parathyroid adenoma. Auris Nasus Larynx 26: 101-104.

16. Sen P, Flower N, Papesch M, Davis A, Spedding AV (2000) A benign parathyroid cyst presenting with hoarse voice. J Laryngol Otol 114: 147-148.

17. Akimoto T, Saito O, Muto S, Hasegawa T, Nokubi M, et al. (2005) A case of thoracic hemorrhage due to ectopic parathyroid hyperplasia with chronic renal failure. $\mathrm{Am} \mathrm{J}$ Kidney Dis 45: e109-e114.

18. Nito T, Miyajima C, Kimura M, Sugasawa M (2007) Parathyroid adenoma causing spontaneous cervical hematoma: a case report. Acta Otolaryngol Suppl 127: 160-163.

19. Roy M, Mazeh H, Chen H, Sippel RS (2013) Incidence and localization of ectopic parathyroid adenomas in previously unexplored patients. World J Surg 37: 102-106.

20. Wilhelm SM, Wang TS, Ruan DT, Lee JA, Asa SL, et al. (2016) The American Association of Endocrine Surgeons Guidelines for definitive management of primary hyperparathyroidism. JAMA Surg 151: 959-968.

21. Stack BC Jr, Tolley NS, Bartel TB, Bilezikian JP, Bodenner D, et al. (2018) AHNS Series: Do you know your guidelines? Optimizing outcomes in reoperative parathyroid surgery: Definitive multidisciplinary joint consensus guidelines of the American Head and Neck Society and the British Association of Endocrine and Thyroid Surgeons. Head Neck 40: 1617-1629. 